\title{
THE DYNAMICS OF RAPID REDSHIFTED AND BLUESHIFTED EXCURSIONS IN THE SOLAR H $\alpha$ LINE
}

\author{
D. Kuridze ${ }^{1,4}$, V. Henriques ${ }^{1}$, M. Mathioudakis ${ }^{1}$, R. Erdélyi ${ }^{2}$, T. V. Zaqarashvilit ${ }^{3,4}$, S. ShelyaG ${ }^{5}$, P. H. Keys ${ }^{1,2}$, and \\ F. P. KEENAN ${ }^{1}$ \\ ${ }^{1}$ Astrophysics Research Centre, School of Mathematics and Physics, Queen's University, Belfast BT7 1NN, UK; d.kuridze@qub.ac.uk \\ ${ }^{2}$ Solar Physics and Space Plasma Research Centre (SP $\left.{ }^{2} \mathrm{RC}\right)$, University of Sheffield, Hicks Building, Hounsfield Road, Sheffield S3 7RH, UK \\ ${ }^{3}$ Space Research Institute, Austrian Academy of Sciences, Schmiedlstrasse 6, A-8042 Graz, Austria \\ ${ }^{4}$ Abastumani Astrophysical Observatory at Ilia State University, 3/5 Cholokashvili avenue, 0162 Tbilisi, Georgia \\ ${ }^{5}$ Monash Centre for Astrophysics, School of Mathematical Sciences, Monash University, Clayton VIC 3800, Australia \\ Received 2014 October 21; accepted 2015 January 23; published 2015 March 17
}

\begin{abstract}
We analyze high temporal and spatial resolution time-series of spectral scans of the $\mathrm{H} \alpha$ line obtained with the CRisp Imaging SpectroPolarimeter instrument mounted on the Swedish Solar Telescope. The data reveal highly dynamic, dark, short-lived structures known as Rapid Redshifted and Blueshifted Excursions (RREs, RBEs) that are on-disk absorption features observed in the red and blue wings of spectral lines formed in the chromosphere. We study the dynamics of RREs and RBEs by tracking their evolution in space and time, measuring the speed of the apparent motion, line of sight (LOS) Doppler velocity, and transverse velocity of individual structures. A statistical study of their measured properties shows that RREs and RBEs have similar occurrence rates, lifetimes, lengths, and widths. They also display non-periodic, nonlinear transverse motions perpendicular to their axes at speeds of 4-31 km s${ }^{-1}$. Furthermore, both types of structures either appear as high speed jets and blobs that are directed outwardly from a magnetic bright point with speeds of $50-150 \mathrm{~km} \mathrm{~s}^{-1}$, or emerge within a few seconds. A study of the different velocity components suggests that the transverse motions along the LOS of the chromospheric flux tubes are responsible for the formation and appearance of these redshifted/blueshifted structures. The short lifetime and fast disappearance of the RREs/RBEs suggests that, similar to type II spicules, they are rapidly heated to transition region or even coronal temperatures. We speculate that the Kelvin-Helmholtz instability triggered by observed transverse motions of these structures may be a viable mechanism for their heating.
\end{abstract}

Key words: magnetohydrodynamics (MHD) - Sun: atmosphere - Sun: chromosphere - Sun: magnetic fields Sun: oscillations - waves

\section{INTRODUCTION}

The highly inhomogeneous nature of the solar chromosphere has been known since the discovery of solar spicules almost $140 \mathrm{yr}$ ago (Secchi 1877). Spicules are small-scale, jet-like plasma features observed ubiquitously at the solar limb between the photosphere and the corona (Roberts 1945; Beckers 1968, 1972; Sterling 2000; Tsiropoula et al. 2012). The traditional (type I) spicules have lifetimes ranging from 1-12 minutes and are characterized by rising and falling motions with speeds of $\sim 20-40 \mathrm{~km} \mathrm{~s}^{-1}$. Subsequent observations have revealed similar types of chromospheric fine structures such as quiet Sun mottles (Suematsu et al. 1995) and active region fibrils on the solar disk. Observations with the Solar Optical Telescope onboard Hinode have identified a different type of spicule (type II), which are more energetic and short-lived features characterized only by upward apparent motions (de Pontieu et al. 2007a).

Another class of fine-scale chromospheric structures, called the Rapid Blueshifted Excursions (RBEs), was found on the solar disk using high resolution, ground-based spectroscopic observations (Langangen et al. 2008). These are absorption features detected in the blue wings of the Ca II $8542 \AA$ and $\mathrm{H} \alpha$ $6563 \AA$ lines (Langangen et al. 2008; Rouppe van der Voort et al. 2009). The measured properties of RBEs are similar to those reported for type II spicules, supporting the idea that they are the same phenomenon viewed from different observational angles, i.e., limb versus disk (Langangen et al. 2008; Rouppe van der Voort et al. 2009). These dynamic events, characterized by a blueshifted spectral line profile, occur near network boundaries mostly in areas where $\mathrm{H} \alpha$ rosettes are located. Rosettes are clusters of elongated $\mathrm{H} \alpha$ mottles expanding radially around a common center over internetwork regions (Zachariadis et al. 2001; Tziotziou et al. 2003; Rouppe van der Voort et al. 2007). However, compared to mottles, RBEs are slender $(\sim 200 \mathrm{~km})$ and short-lived $(\sim 40 \mathrm{~s})$ with higher apparent velocities $\left(\sim 50-150 \mathrm{~km} \mathrm{~s}^{-1}\right)$. Furthermore, RBEs are characterized by rapid fading (within a few seconds) in chromospheric spectral lines without any descending behavior. This rapid disappearance may be the result of fast heating to transition region and coronal temperatures (Rouppe van der Voort et al. 2009). Some recent observational evidence supports the heating hypothesis in limb spicules (de Pontieu et al. 2011; Pereira et al. 2014). However, the mechanism for this fast heating remains a mystery.

Another important dynamical characteristic of these structures is their transverse displacements. Like spicular structures, RBEs undergo transverse motions perpendicular to their longitudinal axis (Rouppe van der Voort et al. 2009). In relatively long-lived structures, such as type I spicules and mottles, these motions are usually observed as periodic transverse displacements, and interpreted as magnetohydrodynamic (MHD) kink waves (Zaqarashvili et al. 2007; Pietarila et al. 2011; Kuridze et al. 2012; Morton et al. 2012; Jess et al. 2012). However, it is extremely difficult to detect any such periodicity in RBEs due to their short lifetime.

Sekse et al. (2013a) reported the presence of RBE-like absorption features in the red wing of the Ca II $8542 \AA$ and $\mathrm{H} \alpha$ 

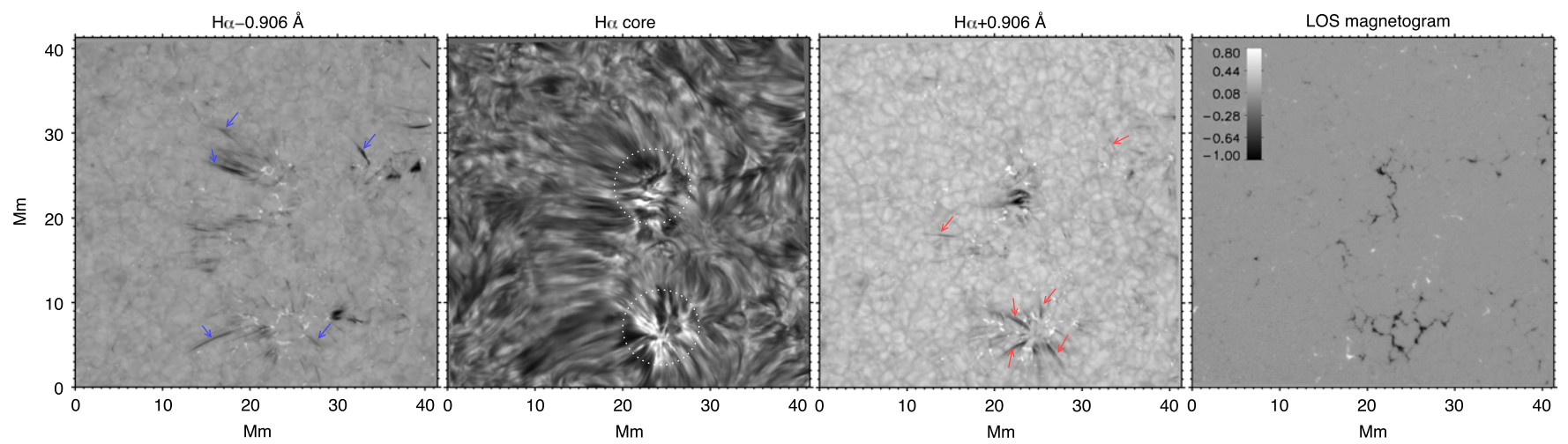

Figure 1. $\mathrm{H} \alpha$ core and $\pm 0.906 \AA$ images together with the photospheric, line of sight magnetogram obtained from Fe $6302 \AA$ Stokes $V$ profiles. Red and blue arrows indicate typical RREs and RBEs observed in the $\mathrm{H} \alpha$ line wings. The rosette regions where most of the RBEs/RREs are detected are highlighted with white dotted circles. The images show that the footpoints of the RBEs/RREs correspond to photospheric bright points and strong magnetic flux concentrations. The color scale in the magnetogram indicates the magnetic field strength in kilogauss.

$6563 \AA$ lines, referred to as Rapid Redshifted Excursions (RREs). They are detected in the same regions, along chromospheric magnetic flux concentrations, where RBEs and $\mathrm{H} \alpha$ mottles are usually observed, but with a lower occurrence rate. Sekse et al. (2013a) suggested that three kinds of motion in the chromospheric flux tube, i.e., field-aligned flows, swaying motions and torsional motions, can result in a net redshift that is manifested as RREs. Lipartito et al. (2014), have reported similar structures in the $\mathrm{H} \alpha$ wing (blue- and redwing fibrils) near the solar limb. These structures occur with comparable occurrence rates without any evidence for torsional motions. Lipartito et al. (2014) have presented arguments that at least some of the detected structures may correspond to warps in two-dimensional sheets, which is an alternative interpretation for dynamic type II spicular-like features (Judge et al. 2011).

Here, we present high spatial and temporal resolution imaging spectroscopy of RREs and RBEs in the $\mathrm{H} \alpha$ on-disk, quiet solar chromosphere. The structures are detected at symmetric wing positions located at $\pm 0.906 \AA$ from the $\mathrm{H} \alpha$ line core. Some of them are high-speed, dark, blob-like features moving upwardly along magnetic flux tubes. To investigate their dynamical characteristics and spatio-temporal evolution we perform detail measurements of their line of sight (LOS) velocities, transverse velocities in the image plane, and apparent velocities of the individual structures. Using the measured properties, the interrelations between RREs and RBEs and the formation of their spectral line asymmetries are investigated. We discuss which mechanism could be responsible for the rapid heating, and hence the observed fast disappearance of these structures, as well as show the presence of similar features in numerical MHD simulations of the solar atmosphere.

\section{OBSERVATIONS AND DATA REDUCTION}

Observations were undertaken between 09:06 and 09:35 UT on 2013 May 5 at disk center, on the edge of a coronal hole, with the CRisp Imaging SpectroPolarimeter (CRISP; Scharmer 2006; Scharmer et al. 2008) instrument, mounted on the $1 \mathrm{~m}$ Swedish Solar Telescope (Scharmer et al. 2003). The dataset includes spectral imaging in the $\mathrm{H} \alpha 6563 \AA$ and Fe $6302 \AA$ lines. Adaptive optics were used throughout the observations consisting of a tip-tilt mirror and an 85-electrode deformable mirror setup that is an upgrade of the system described in Scharmer et al. (2003). This system provided a near constant "lock" and all reconstructed frames benefited from the adaptive-optics correction.

All data were reconstructed with Multi-object Multi-frame Blind Deconvolution (MOMFBD; van Noort et al. 2005; Löfdahl 2002), using 51 Karhunen-Loève modes sorted by order of atmospheric significance and using $88 \times 88$ pixel subfields in the red. A prototype of the code published by de la Cruz Rodríguez et al. (2014) was used before and after MOMFBD. Reflectivity fitting, due to the broadness of $\mathrm{H} \alpha$, was not used as justified in de la Cruz Rodríguez et al. (2013). The images, reconstructed from the narrowband wavelengths, were aligned at the smallest scales by using the method described by Henriques et al. (2013). This employs crosscorrelation between auxiliary wide-band channels, obtained from an extended MOMFBD scheme, to account for different residual small-scale seeing distortions and different reconstruction PSFs. Data were composed into time-series having been de-rotated, aligned, and finally destretched as in Shine et al. (1994).

Spatial sampling is 0.0592 pixel $^{-1}$ and the spatial resolution up to 0.16 in $\mathrm{H} \alpha$ over the field of view (FOV) of $41 \times 41 \mathrm{Mm}$. The $\mathrm{H} \alpha$ line scan consists of seven positions $(-0.906,-0.543$, $-0.362,0.000,0.362,0.543,+0.906 \AA$ from line core) corresponding to a velocity range of -41 to $+41 \mathrm{~km} \mathrm{~s}^{-1}$ in velocity. Following reconstruction, the cadence of a full spectral scan was $1.34 \mathrm{~s}$. CRISP also undertook photospheric Fe 6301 and $6302 \AA$ spectral scans with 16 wavelength positions per line every $\sim 5$ minutes over the same FOV to obtain full Stokes profiles. The Stokes $V$ component was used to construct LOS magnetograms employing the center of gravity method (Rees \& Semel 1979; Uitenbroek 2003).

The widget-based CRIsp SPectral EXplorer (Vissers \& van der Voort 2012) and Timeslice ANAlysis tools were used to perform the data analysis and the measurements presented in this study.

\section{ANALYSIS AND RESULTS}

Figure 1 shows images in the $\mathrm{H} \alpha$ line core and $\pm 0.906 \AA$ from line core together with the photospheric LOS magnetogram obtained from the $\mathrm{Fe} 6302$ A Stokes $V$ profiles. The $\mathrm{H} \alpha$ line core image contains two large rosette structures, highlighted with white dotted circles (second panel of Figure 1), where most of the chromospheric flux tubes, and hence the 

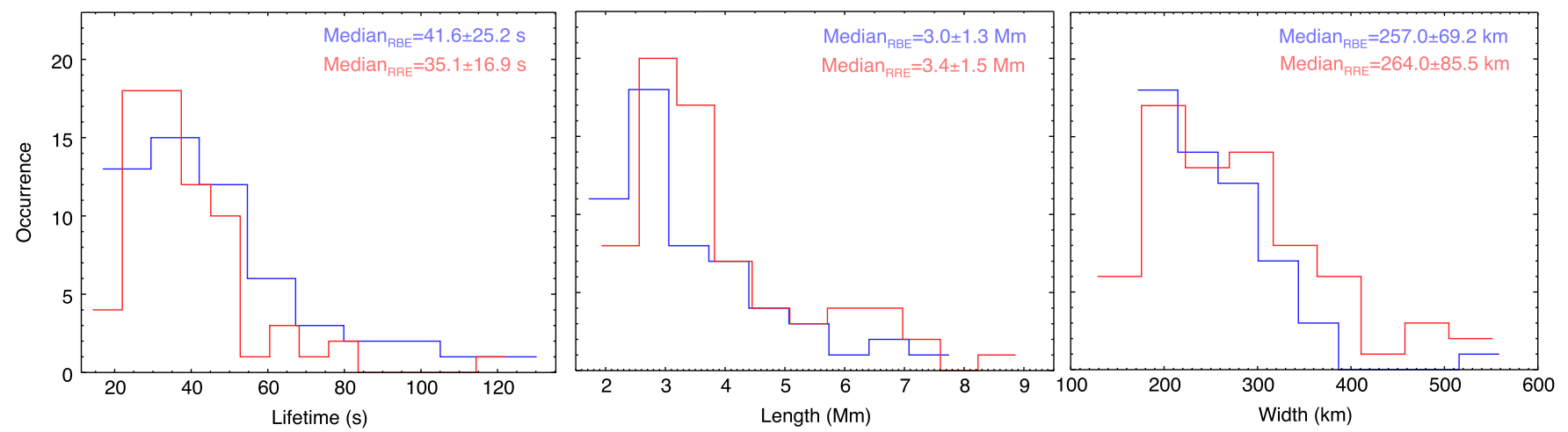

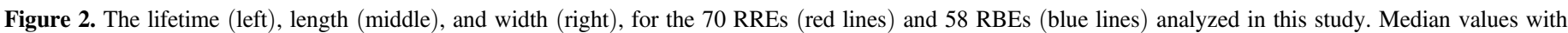
standard deviations are also given.

fine-scale structures, are concentrated. The roots of the rosette structures are co-spatial with strong magnetic field concentrations that outline the intergranular lanes. The LOS magnetogram shows that the FOV consists mostly of unipolar patches with magnetic field strength in the kilogauss range.

We selected dark absorption features manually (i.e., by visual inspection) in the far red and blue wings at $\mathrm{H} \alpha \pm 0.906 \AA$ that are longer than $1.5 \mathrm{Mm}$ for at least $10 \mathrm{~s}$, and have an aspect ratio (length/width) greater than $\sim 5$ at maximum extension. We have excluded granular boundaries and so-called swirls, characterized by vortex-like behavior, from our selection. Based on these criteria, we detected 70 features in the far red wing at $\mathrm{H} \alpha+0.906 \AA$ and 58 features in the far blue wing at $\mathrm{H} \alpha-0.906 \AA$, identified as RREs and RBEs respectively. Examples of these dark absorbing structures, originating from magnetic bright points along chromospheric flux tubes, are indicated by the red and blue arrows in the $\mathrm{H} \alpha$ line wing images (Figure 1). It should be noted that the number of RBEs/ RREs detected in this work is much smaller than the number of detections by an automated algorithm used by other authors (see e.g., Sekse et al. 2012). However, our selection is restricted to features that are clearly distinguishable, fully observable from edge to edge, and fully traceable in time in the far wing images of the $\mathrm{H} \alpha$ time sequence. This reduces the number of detections but gives us a more robust dataset to investigate the different velocity components and the spatialtemporal evolution which is the ultimate goal of this work. We also want to note that the total number of detected RREs is higher than the number of RBEs (70 versus 58), we believe that RREs and RBEs appear here with a similar occurrence rate. The difference in numbers may be attributed to the lower contrast between the background and RBEs.

We have measured each structure's lifetime as the time interval between its first appearance and complete disappearance; the maximum projected length as the maximum visible extension with manually identified lower and upper ends of the structure during its lifetime; the width as the visible diameter of the central part of the detected structure at the time of maximum extension. The width measured with this method agrees very well with FWHM of Gaussian-fit trials performed on the cross-section of the flux profiles.

The results show that RREs and RBEs have almost identical lifetimes, widths, and lengths (Figure 2). Despite these similarities, the analysis does not show that the appearance or disappearance of RREs is correlated with the appearance or disappearance of RBEs, suggesting that they are independent features. Similarly, there is no one-to-one relation between them and $\mathrm{H} \alpha$ line core mottles.

The detected structures either appear as upward-directed high speed jets and blobs, or emerge suddenly within a few time resolution elements $(\sim 1-5 \mathrm{~s})$. Many of them (13 RREs and 22 RBEs) display transverse motions perpendicular to their axis in the image plane. At the end of their lifetime the structures disappear extremely quickly without any apparent downward motion. We note that RREs/RBEs detected in the $\pm 0.906 \AA$ images are normally also visible in neighboring $\pm 0.543 \AA$ images. They appear and disappear simultaneously in both wavelength positions. Transverse velocities and transverse displacements are measured using time-distance $(t-d)$ diagrams generated along the cuts perpendicular to the structure's axes. Figure 3 illustrates a typical example of transverse displacement of a selected RBE. We do not see periodic behavior for the detected transverse motions (i.e., there are no swings from side to side). However, it should be noted that the structures disappear in the $\mathrm{H} \alpha$ images before the transverse motions come to a halt. Transverse velocities are in the range of 4-22 $\mathrm{km} \mathrm{s}^{-1}$, with displacements between 250 and $1200 \mathrm{~km}$ (Figure 4). It is clear that the transverse displacements are greater than the width of the structures $(\sim 250 \mathrm{~km})$, implying that the motions are strongly nonlinear.

The apparent velocities of the RREs and RBEs projected on the image plane are determined using $t-d$ plots, generated along the path of these motions. Velocity estimates are in the range $50-150 \mathrm{~km} \mathrm{~s}^{-1}$ (Figure 4) which are Alfvénic and superAlfvénic in the chromosphere. The apparent absolute velocities of RREs and RBEs are in a similar range, although the numbers of structures where reliable measurements were possible are different (36 versus 5 for RREs and RBEs, respectively). We believe that the higher contrast of the RREs makes the apparent motions in them more easily measurable than in RBEs. The $\mathrm{H} \alpha$ data presented here show no evidence for any downward motions either in the RBEs or in the RREs.

Some of the detected RREs/RBEs appear as high-speed blobs moving upwardly along the same path where other chromospheric fine structures are detected. Figure 5 shows the temporal evolution of a typical dark blob detected in the red wing at $\mathrm{H} \alpha+0.906 \AA$. The blob starts from the bright point and moves upward along a curved trajectory with an apparent propagation speed of $\sim 110 \mathrm{~km} \mathrm{~s}^{-1}$.

The large red and blue line profile asymmetry, which is the main characteriztic of RREs and RBEs, means that they have a LOS velocity component. Values of LOS velocities for each 

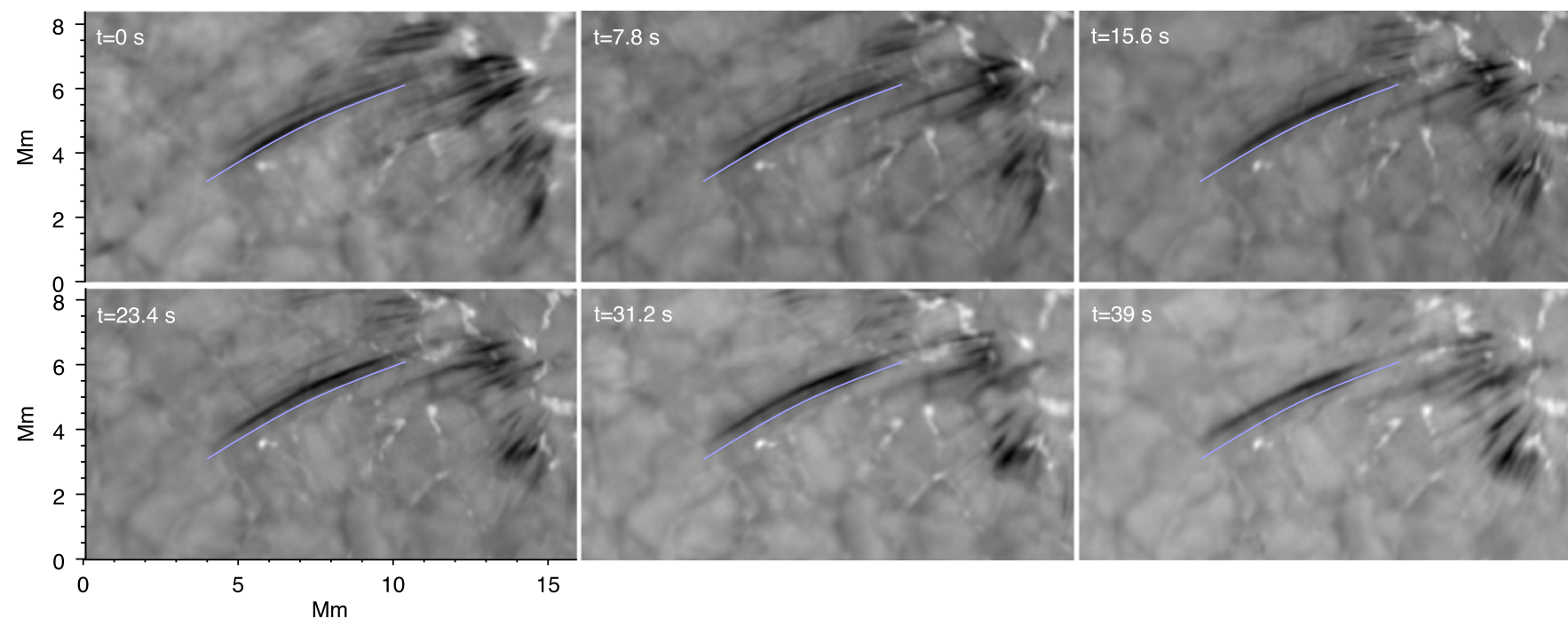

Figure 3. A typical example of the non-periodic transverse displacement of an RBE with a displacement of $\sim 500 \mathrm{~km}$ and a transverse velocity of $\sim 13 \mathrm{~km} \mathrm{~s}{ }^{-1}$. Blue lines show the initial position of the structure for reference.
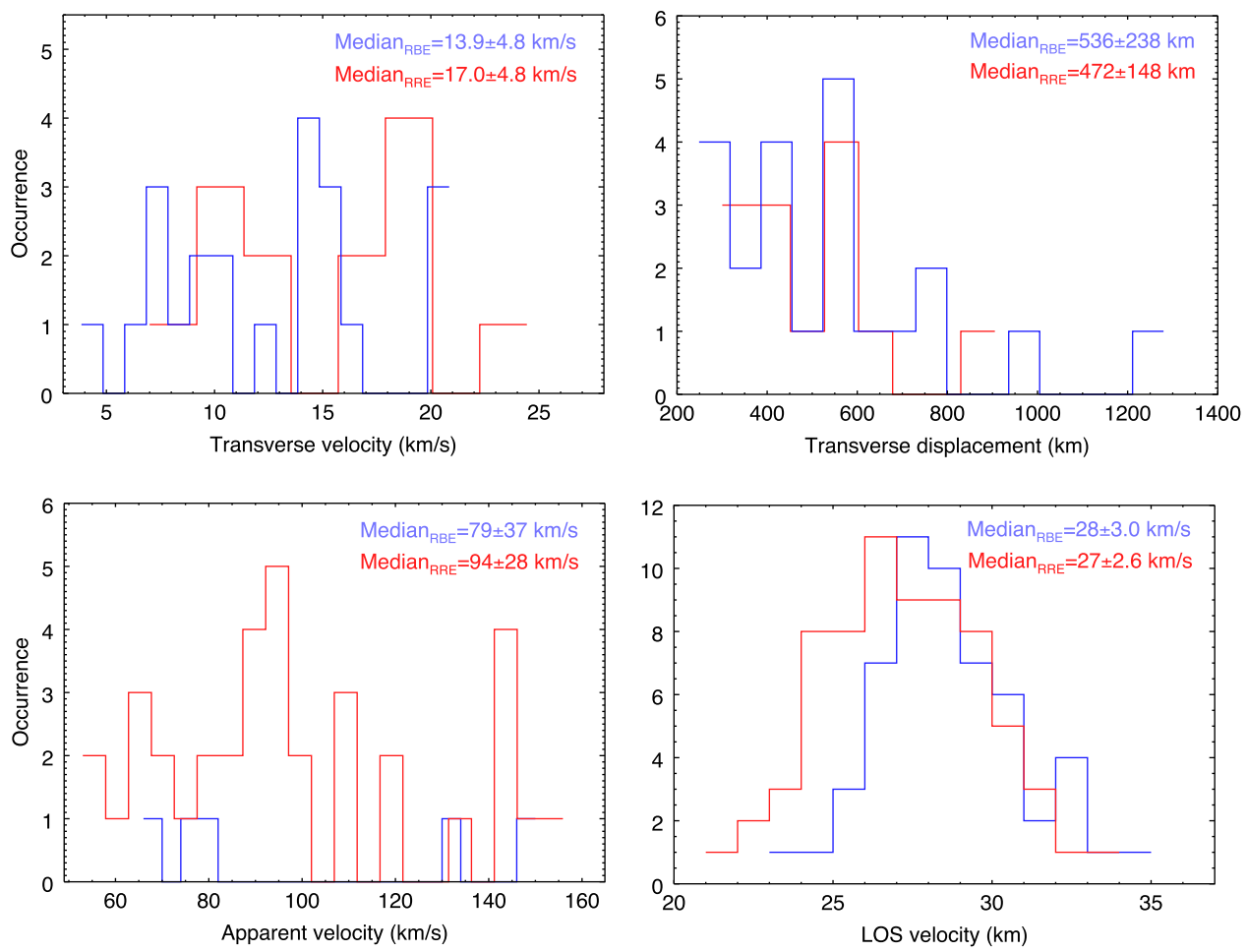

Figure 4. Distribution of the average LOS velocity (absolute value), apparent velocity, transverse velocity (in the image plane) and transverse displacement amplitude for the analyzed RREs and RBEs (red and blue lines, respectively). Median values with standard deviations are also given.
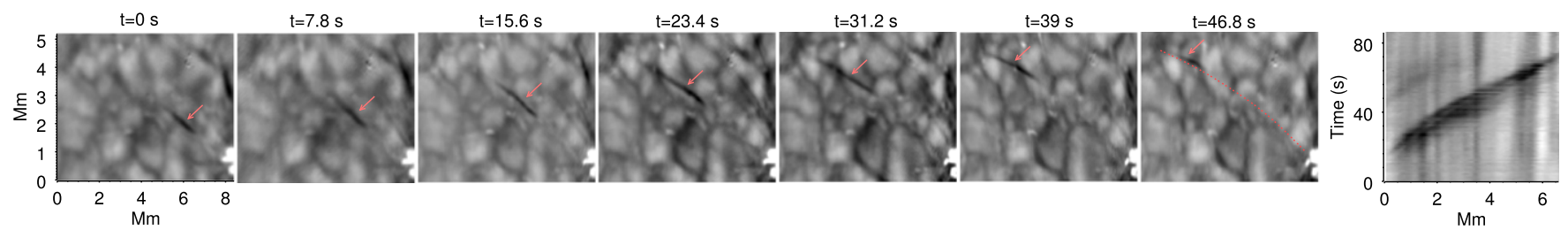

Figure 5. Time-sequence of a typical dark blob detected in the red wing at $\mathrm{H} \alpha+0.906 \AA\left(42 \mathrm{~km} \mathrm{~s}^{-1}\right)$. The blob starts from the network brightening and moves upward along the curved trajectory highlighted with the red dotted line. The corresponding $t-d$ diagram (right panel) estimates an apparent propagation speed of $\sim 110 \mathrm{~km} \mathrm{~s}^{-1}$.

individual feature are calculated using Doppler signals provided by their $\mathrm{H} \alpha$ spectral profile. For these measurements we use the first moment with respect to wavelength method described by Rouppe van der Voort et al. (2009). A box is defined for each manually detected RRE/RBE that covers the whole structure when it has maximum extension of \pm 5 pixels, 
and calculate the $v_{\mathrm{LOS}}(x, y, i)$, where $x$ and $y$ are transverse and longitudinal coordinates of this box, and $i=j, \ldots, k$ so that $j$ and $k$ are the number of frames where the structure first appears and disappears completely, respectively. Doppler velocities were compensated after computation for etalon cavity errors. Our analysis shows that the LOS velocities are approximately constant along the lengths of RREs/RBEs. Furthermore, they are almost regular and do not show any type of periodic behavior over their lifetime. Absolute values of these velocities, averaged along the structures lengths, are in the range of $21-34 \mathrm{~km} \mathrm{~s}^{-1}$. The distribution of LOS velocities is shown in Figure 4 (bottom right panel) where again both types of structures have similar LOS velocities.

We inspected the locations where RBEs or RREs were detected a few minutes before and after the appearance/ disappearance of each structure and looked for evidence in both the LOS Doppler velocity signal and intensity in the line wings. We found no significant signal and therefore no evidence of returning or periodic motions.

\section{DISCUSSION}

\subsection{Transverse Displacements}

A wide range of transverse motions have been studied thoroughly in chromospheric fine structures such as type I and II spicules (Zaqarashvili et al. 2007; Zaqarashvili \& Erdélyi 2009; Okamoto \& de Pontieu 2011), mottles (Kuridze et al. 2012, 2013; Morton et al. 2012), fibrils (Pietarila et al. 2011), and RBEs (Rouppe van der Voort et al. 2009; Sekse et al. 2013a). These motions frequently reveal periodic behavior which is attributed to MHD kink waves (Spruit 1982). Reported wave periods in the relatively long-lived chromospheric features, such as type I spicules, mottles and fibrils, are in the range 1-5 minutes. The mean period of the kink waves in the $\mathrm{H} \alpha$ line core mottles is $\sim 3$ minutes (Kuridze et al. 2012; Morton et al. 2012). RREs/RBEs have similar geometries and spatial scales (length and width) as the $\mathrm{H} \alpha$ mottles. Furthermore, as they occur at the same place and their footprints are almost co-spatial, it may be expected that they will have the same wave drivers. Thus waves with similar periods may be expected to be excited in them as well. However, RREs/RBEs are short-lived, compared to mottles, with mean lifetimes much lower than the typical periods of transverse kink waves in chromospheric fine structures. This makes it extremely challenging to determine the periodicity of these motions in RREs and RBEs. We note that some evidence of periodic transverse motions in RBEs has been reported by Sekse et al. (2013b). The transverse motions analyzed in this work do not show full swings (i.e., motions from left to right and back again) that would be the manifestation of kink waves. RREs and RBEs display a nonlinear transverse motion in only one direction that does not come to a halt before the structure disappears. This suggests that the reason for the lack of a full wave cycle is due to the relatively short lifetimes of the RREs and RBEs. It should be noted that the strong damping of these motions could be an alternative explanation for their nonperiodic nature. However, scaling laws of existing damping models, such as e.g., resonant absorption, phase mixing, wave leakage or mode conversion, indicate that the damping timescales should be longer than the wave periods (Goossens et al. 2002, 2014; Ofman \& Aschwanden 2002). This suggests that a periodicity could still have been seen. It could be argued that the observed transverse motions are not waves, but just transverse displacement of the flux tube as a whole from its initial position. Hasan \& Kalkofen (1999) have shown that when transverse motions are excited by a photospheric granule, and the interaction timescale between the granule and the flux tube is comparable to or greater than the chromospheric cutoff period, there is no kink wave. Instead, the whole tube is simply displaced from its initial location at all heights. Unfortunately, the dataset analyzed in this work does not allow us to determine the excitation mechanisms, and hence the exact nature of the detected transverse motions.

\subsection{Apparent and LOS Velocities}

The observations presented here show that RREs and RBEs either display apparent motions that are directed outwardly from the magnetic bright point, or emerge suddenly within a few seconds. Our very high cadence $(1.3 \mathrm{~s})$ allowed us to estimate apparent motions for some of the RREs and RBEs with speeds ranging between $50-150 \mathrm{~km} \mathrm{~s}^{-1}$ (Figure 4). Similar behavior in RREs and type II spicules has been reported by other authors (Rouppe van der Voort et al. 2009; Sekse et al. 2013a). The Alfvénic/super-Alfvénic apparent velocities measured here, and the sudden appearance of the structures, are their most puzzling aspects. One possible explanation is that they are formed as a result of upward directed plasma flows along chromospheric flux tubes. A number of recent numerical simulations have shown that the high-speed upflow patterns in the chromosphere can be generated by photospheric vortex motions in magnetic features and Lorentz forces (Goodman 2012; Kitiashvili et al. 2013). However, the extremely fast appearance (with speeds of more than $\sim 700 \mathrm{~km} \mathrm{~s}^{-1}$ ) is still unexplained by this flow scenario. Furthermore, it does not explain the appearance of large numbers of redshifted features (RREs) at the solar disk center. The sources of the Doppler signals of the chromospheric flux tube could be field-aligned flows and/or the transverse motions along the LOS. It must be noted that torsional motions can also contribute to the line shift, but we have not found any evidence of torsional motions in our dataset. Field-aligned flows that appear from the bright points and move outwardly along a magnetic flux tube can produce blueshifted Doppler signals, $v_{\text {dopp }} \rightarrow$ blue, if the tilt angle $(\theta)$ with respect to the observer is $<90^{\circ}, v_{\text {dopp }} \rightarrow 0$ if $\theta \approx 90^{\circ}$, and $v_{\text {dopp }} \rightarrow$ red if $\theta>90^{\circ}$. As the observed quiet Sun region is almost unipolar (right panel of Figure 1) it is expected that the region is dominated by open flux tubes, which suggests that they do not have $\theta<90^{\circ}$ at the disk center. Thus, flows can only produce blueshifted signals whereas transverse motions can generate both red and blue Doppler-shifts without any preference. Hence, if the apparent motions are plasma flows, then there are more sources of blueshifted Doppler signals. There should therefore be a prevalence of RBEs and the absolute values of the Doppler velocities for the RBEs should be higher than those of RREs. However, we could find no evidence that would substantiate this scenario. By contrast, the number of detected RREs is higher than that of RBEs and they have similar absolute values of LOS velocities (bottom right panel of Figure 4). This indicates that field-aligned flows is an unlikely explanation for the fast appearance of the RREs and RBEs.

An alternative interpretation for the high apparent velocities and rapid, sudden appearance of these features is based on a wave scenario. The basic idea is that upward propagating kink 
waves/disturbances along the flux tube can be seen as Doppler signals in the form of RREs/RBEs appearing with Alfvénic speed. Indeed, the observed high-speed redshifted/blueshifted jet- and blob-like features may be interpreted in terms of propagating transverse pulses/disturbances traveling with a phase speed which could be close to the chromospheric Alfvén speed. On the other hand, superposition of the oppositedirected kink waves may result in a wave with a very high/ infinite phase speed (standing waves) that can be manifested as the sudden appearance of RREs/RBEs. Both propagating (upward and downward) and standing, transverse oscillations have been reported in type II spicules at the limb (Okamoto \& de Pontieu 2011) and mottles on the disk (Kuridze et al. 2013). However, there is no evidence of downward propagating signals in RBEs and RREs. Furthermore, as noted by Lipartito et al. (2014), standing waves require a finite time to set up. This time is estimated as $t \sim l / v_{A} \mathrm{~s}$ (Judge et al. 2012), where $l$ and $v_{A}$ are the structure's length and chromospheric Alfvén speed, respectively. RREs and RBEs have typical lengths of $\sim 3 \mathrm{Mm}$, and $v_{A}$ is estimated as $\sim 20-80 \mathrm{~km} \mathrm{~s}^{-1}$ in the chromosphere. Hence $t$ is $\sim 40-150 \mathrm{~s}$ which is comparable to greater than their typical lifetime $(\sim 40 \mathrm{~s})$. It is therefore doubtful that standing wave patterns, generated by the superposition of oppositely directed waves, are responsible for the observed fast appearance of the RREs/RBEs.

The main aspect of the reported transverse motions in RREs and RBEs is that the structure is displaced as a whole, i.e., every single part of their visible length is moved in phase, in the same direction and with the same speed. These motions $\left(v_{\operatorname{tr}_{\perp}}\right)$ are detected in the image plane. However, it is expected that the motions should also have a LOS component $\left(v_{\operatorname{tr}_{\mathrm{LOS}}}\right)$ and some should also be polarised in the LOS plane. If the flux tubes start to move along the LOS in a similar way to what is detected in the image plane (Figure 3), then they should appear suddenly in the $\mathrm{H} \alpha$ line wings as redshifted or blueshifted features depending on the direction of the LOS velocity with respect to the observer. We propose that the observed RREs and RBEs are manifestations of the chromospheric flux tubes' transverse motions along the LOS. Similar to the transverse motions in the image plane, LOS Doppler signals and thus the associated transverse motions do not show any evidence of periodic behavior, e.g., periodic variation or the change of the flux tubes' Doppler-shift sign. It must be noted that the obtained median values of $v_{\text {troOs }}\left(\sim 27-28 \mathrm{~km} \mathrm{~s}^{-1}\right.$ for RREs and RBEs, respectively) are higher than the median of $v_{\operatorname{tr}_{\perp}}$ ( $\sim 14-17 \mathrm{~km} \mathrm{~s}^{-1}$ for RREs and RBEs, respectively). However, as the analyzed structures are detected at the far red and blue wing positions of $\mathrm{H} \alpha \quad( \pm 0.906 \AA$ corresponding to $\left.\pm 41 \mathrm{~km} \mathrm{~s}^{-1}\right), v_{\text {tr }_{\text {LOS }}}$ derived from spectroscopic observations are biased toward detecting higher velocity events, which may be the reason for the discrepancy between the average values of $v_{\operatorname{tr}_{\mathrm{LOS}}}$ and $v_{\mathrm{tr}_{\perp}}$.

\subsection{Stability}

Transverse motions of the RREs/RBEs can create velocity discontinuities between the surface of the flux tube and surrounding media, which may trigger the Kelvin-Helmholtz Instability (KHI). The component of the magnetic field parallel to the discontinuity tends to stabilize sub-Alfvénic flows (Chandrasekhar 1961), while the perpendicular component has no effect on KHI (Sen 1963). This suggests that the tube moving with any speed at an angle to the magnetic field is unstable to KHI for sufficiently high azimuthal wave number $m$ (Zaqarashvili et al. 2014). The observed velocities of the LOS transverse motions of the RREs/RBEs $\left(\sim 21-34 \mathrm{~km} \mathrm{~s}^{-1}\right)$ could be higher than the local Alfvén speed, which may significantly enhance the growth rate of KHI.

The growth rate of KHI of a transversally moving magnetic structure can be easily estimated in the case of slab geometry. The dispersion relation of antisymmetric incompressible perturbations for a magnetic slab of half-width $d$ moving transversally in its plane can be written as (Zaqarashvili et al. 2011)

$$
\begin{gathered}
\rho_{0}\left[(\omega-\boldsymbol{k} \cdot \boldsymbol{U})^{2}-\left(\boldsymbol{k} \cdot \boldsymbol{V}_{A 0}\right)^{2}\right] \operatorname{coth}(k d) \\
+\rho_{e}\left[\omega^{2}-\left(\boldsymbol{k} \cdot \boldsymbol{V}_{A e}\right)^{2}\right]=0
\end{gathered}
$$

where $\rho_{0}, \rho_{e}$, and $\boldsymbol{V}_{A 0}, \boldsymbol{V}_{A e}$ are the densities and Alfvén speeds inside and outside the slab, respectively, $\boldsymbol{U}$ is the unperturbed velocity (transverse velocity of flux tube in our case) and $\boldsymbol{k}$ is the wave vector. For symmetric perturbations $\operatorname{coth}(k d)$ should be replaced by $\tanh (k d)$. For $|k d|>1$, both coth $(k d)$ and $\tanh (k d)$ are constant and equal to unity. Then Equation (1) is simplified and using $\boldsymbol{k} \cdot \boldsymbol{V}_{A 0}=\boldsymbol{k} \cdot \boldsymbol{V}_{A e} \approx 0$ we get the imaginary part of perturbations, which is actually the growth rate, as $\omega_{i} \approx k U \sqrt{\rho_{e} / \rho_{0}}$. Thus the growth time of KHI depends on the transverse velocity, the density contrast, and the spatial scale of perturbations. A transverse velocity of $20 \mathrm{~km} \mathrm{~s}^{-1}$, a spatial scale of the order of tube width of $200 \mathrm{~km}$, and the density contrast of $\rho_{e} / \rho_{0}=0.1$ lead to the growth time of $5 \mathrm{~s}$.

The generated KH vortices may lead to enhanced MHD turbulence near the tube boundaries through nonlinear cascade. Shear flow energy then can be rapidly transferred to small scales, where it will dissipate and heat the surrounding plasma. This explains the fast heating of the chromospheric flux tubes and hence the observed sudden disappearance of RREs/RBEs. However, this is a speculative interpretation and detailed numerical simulations are required to assess the importance of KHI to the stability of these features.

\subsection{Numerical Modeling}

We carried out numerical modeling using the radiative MHD code MuRAM (Vögler et al. 2005). In a numerical domain, which covers the top $2 \mathrm{Mm}$ of the convection zone and the bottom 1.2 Mm of the solar atmosphere, transparent bottom and semi-transparent (allowing outflows) top boundary conditions were used. A non-magnetic convection snapshot was employed as an initial configuration, where $200 \mathrm{G}$ uniform unipolar vertical magnetic field was injected. The numerical domain was then let to develop for about 20 minutes of physical time. One of the developed snapshots of the simulation was used to carry out $\mathrm{H} \alpha$ diagnostics with the NICOLE code in non-LTE (SocasNavarro et al. 2014). The diagnostics showed elongated features similar to RBEs with lifetimes of $\sim 40 \mathrm{~s}$ and a length of few Mm, naturally appearing in the simulations (see Figure 6, first panel). These features are produced by strong, short-lived localized plasma motions upward along the field lines of magnetic flux concentrations in the low- $\beta$ plasma (Figure 6, second panel). The RBEs observed in the simulations do not appear aligned with the magnetic field, however they lie at constant Alfvénic surfaces and are possible signatures of dissipation of torsional Alfvén waves (Shelyag et al. 2013; Shelyag \& Przybylski 2014, also Figure 6, third 

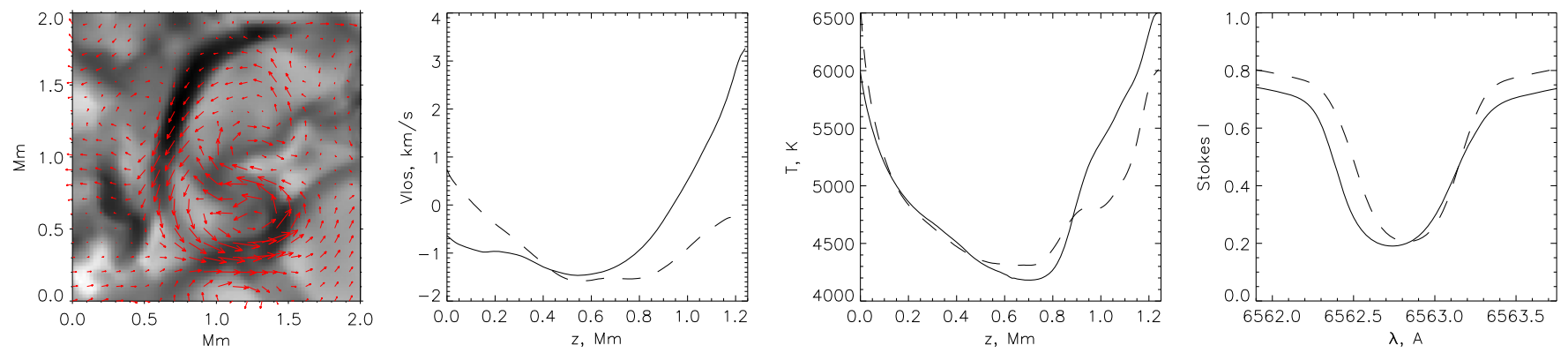

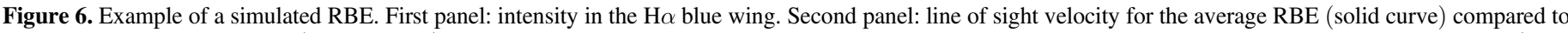

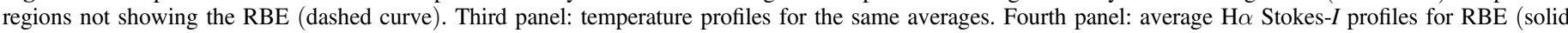

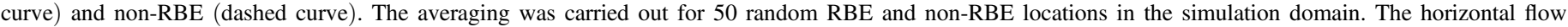
structure around the simulated RBE is shown with arrows in the first panel.

panel). Despite some qualitative similarities between the observations and the simulated $\mathrm{H} \alpha$ line profiles (see Figure 6, fourth panel) as well as their temporal and spatial characteristics, it is still not fully clear if the features seen in the simulations are the observed RBEs. There is a significant difference between the shape of simulated and observed RBEs, which may be a result of forcing the magnetic field to be vertical at the top of the simulation domain. However, if it is assumed that the observed RBE structures track the field lines in strongly inclined (relative to the LOS) magnetic field concentrations, the torsional velocity component would cause the red- and blueshift of the $\mathrm{H} \alpha$ profile. A torsional velocity component is present in the flux tubes in the simulations (Figure 6, first panel) and is linked to Alfvén waves propagating from the base of the photosphere (Shelyag et al. 2013). Furthermore, it should be noted that no strong RREs are observed in the model due to the top boundary condition in the MHD simulation, which does not permit downflows across the boundary, while the outflows (and, correspondingly, RBEs) are allowed.

\section{CONCLUDING REMARKS}

We present a detailed study of the temporal evolution of RREs and RBEs and their velocity components. The latter suggest that the transverse motions along the LOS are responsible for their formation and sudden appearance in the $\mathrm{H} \alpha$ line wings. RREs and RBEs that appear in the form of jets and blobs with Alfvénic velocities may be interpreted as transverse pulses/disturbances propagating along magnetic flux tubes. We propose that the transverse motions of chromospheric flux tubes can develop the KHI at the tube boundaries, which may lead to the rapid heating and sudden disappearance of RREs/RBEs. Numerical MHD modeling shows features qualitatively similar to the observed RBE. However, further study is necessary to provide an unambiguous link between the simulated and observed events. A more detailed study of the simulated RBEs at different solar disk positions and angles between the magnetic field direction and the LOS is required.

The research leading to these results has received funding from the European Community's Seventh Framework Programme (FP7/2007-2013) under grant agreement no. 606862 (F-CHROMA). This research was supported by the SOLARNET project (www.solarnet-east.eu), funded by the European Commissions FP7 Capacities Program under the Grant Agreement 312495. The work of T.Z. was supported by FP7-
PEOPLE-2010-IRSES-269299 project- SOLSPANET, by Shota Rustaveli National Science Foundation grant DI/14/ 6-310/12, and by the Austrian Fonds zur Frderung der wissenschaftlichen Forschung under project P26181-N27. Dr Shelyag gratefully thanks Centre for Astrophysics \& Supercomputing of Swinburne University of Technology (Australia), NCI National Facility systems at the Australian National University, supported by Astronomy Australia Limited, and the Multi-modal Australian ScienceS Imaging and Visualisation Environment (MASSIVE) for the computational resources provided. Dr Shelyag is the recipient of an Australian Research Councils Future Fellowship (project number FT120100057).

\section{REFERENCES}

Beckers, J. M. 1968, SoPh, 3, 367

Beckers, J. M. 1972, ARA\&A, 10, 73

Chandrasekhar, S. 1961, Hydrodynamic and Hydromagnetic Stability (Dover Classics of Science and Mathematics; New York: Dover)

de la Cruz Rodríguez, J., Rouppe van der Voort, L., Socas-Navarro, H., \& van Noort, M. 2013, A\&A, 556, A115

de la Cruz Rodríguez, J., Lofdahl, M., Hillberg, T., et al. 2014, arXiv 1406.0202

de Pontieu, B., McIntosh, S., Hansteen, V. H., et al. 2007a, PASJ, 59, 655 de Pontieu, B., McIntosh, S. W., Carlsson, M., et al. 2011, Sci, 331, 55

Goodman, M. L. 2012, ApJ, 757, 188

Goossens, M., Andries, J., \& Aschwanden, M. J. 2002, A\&A, 394, L39

Goossens, M., Soler, R., Terradas, J., van Doorsselaere, T., \& Verth, G. 2014, ApJ, 788, 9

Hasan, S. S., \& Kalkofen, W. 1999, ApJ, 519, 899

Henriques, V. M. J. 2013, PhD thesis, Stockholm Univ.

Jess, D. B., Pascoe, D. J., Christian, D. J., et al. 2012, ApJ, 744, L5

Judge, P. G., Tritschler, A., \& Low, B. C. 2011, ApJ, 730, L4

Judge, P. G., Reardon, K., \& Cauzzi, G. 2012, ApJL, 755, L11

Kitiashvili, I. N., Kosovichev, A. G., Lele, S. K., Mansour, N. N., \& Wray, A. A. 2013, ApJ, 770, 37

Kuridze, D., Morton, R. J., Erdélyi, R., et al. 2012, ApJ, 750, 51

Kuridze, D., Verth, G., Mathioudakis, M., et al. 2013, ApJ, 779, 82

Langangen, Ø., de Pontieu, B., Carlsson, M., et al. 2008, ApJL, 679, L167

Lipartito, I., Judge, P. G., Reardon, K., \& Cauzzi, G. 2014, ApJ, 785, 109

Löfdahl, M. G. 2002, Proc. SPIE, 4792, 146

Morton, R. J., Verth, G., Jess, D. B., et al. 2012, NatCo, 3, 1315

Ofman, L., \& Aschwanden, M. J. 2002, ApJ, 576, L153

Okamoto, Takenori J., \& de Pontieu, B. 2011, ApJ, 736, L24

Pereira, T. M. D., de Pontieu, B., Carlsson, M., Hansteen, V., et al. 2014, ApJ, 792, L15

Pietarila, A., Aznar Cuadrado, R., Hirzberger, J., \& Solanki, S. K. 2011, ApJ, 739, 92

Rees, D. E., \& Semel, M. D. 1979, A\&A, 74, 1

Roberts, W. O. 1945, ApJ, 101, 136

Rouppe van der Voort, L., De Pontieu, B., Hansteen, V., Carlsson, M., \& van Noort, M. 2007, ApJ, 660, 169

Rouppe van der Voort, L., Leenaarts, J., de Pontieu, B., Carlsson, M., \& Vissers, G. 2009, ApJ, 705, 272 
Scharmer, G. B., Bjelksjo, K., Korhonen, T. K., Lindberg, B., \& Petterson, B. 2003, Proc. SPIE, 4853, 341

Scharmer, G. B. 2006, A\&A, 447, 1111

Scharmer, G. B., Narayan, G., Hillberg, T., et al. 2008, ApJ, 689, L69

Secchi, P. A. 1877, Le Soleil, Vol. 2, (Paris: Gauthier-Villars)

Sekse, D. H., Rouppe van der Voort, L., \& de Pontieu, B. 2012, ApJ, 752, 108

Sekse, D. H., Rouppe van der Voort, L., de Pontieu, B., \& Scullion, E. 2013a, ApJ, 769, 44

Sekse, D. H., Rouppe van der Voort, L., \& de Pontieu, B. 2013b, ApJ, 764, 164 Sen, A. K. 1963, PhFl, 6, 1154

Shelyag, S., Cally, P. S., Reid, A., \& Mathioudakis, M. 2013, ApJL, 776, L4 Shelyag, S., \& Przybylski, D. 2014, PASJ, 66, 9

Shine, R. A., Title, A. M., Tarbell, T. D., et al. 1994, ApJ, 430, 413

Socas-Navarro, H., de la Cruz Rodriguez, J., Asensio Ramos, A., Trujillo Bueno, J., \& Ruiz Cobo, B. 2014, arXiv:1408.6101

Spruit, H. C. 1982, SoPh, 75, 3

Sterling, A. C. 2000, SoPh, 196, 79
Suematsu, Y., Wang, H., \& Zirin, H. 1995, ApJ, 450, 411

Tsiropoula, G., Tziotziou, K., Kontogiannis, I., et al. 2012, SSRv, 169, 181

Tziotziou, K., Tsiropoula, G., \& Mein, P. 2003, A\&A, 402, 361

Uitenbroek, H. 2003, ApJ, 592, 1225

van Noort, M., Rouppe van der Voort, L., \& Löfdahl, M. G. 2005, SoPh, 228, 191

Vissers, G., \& Rouppe van der Voort, L. 2012, ApJ, 750, 22

Vögler, A., Shelyag, S., Schüssler, M., et al. 2005, A\&A, 429, 335

Zachariadis, Th. G., Dara, H. C., Alissandrakis, C. E., Koutchmy, S., \& Contikakis, C. 2001, SoPh, 202, 41

Zaqarashvili, T. V., Khutsishvili, E., Kukhianidze, V., \& Ramishvili, G. 2007, A\&A, 474, 627

Zaqarashvili, T. V., \& Erdélyi, R. 2009, SSRv, 149, 355

Zaqarashvili, T. V. 2011, in AIP Conf. Proc. 1356, 3rd School and Workshop on Space Plasma Physics, ed. I. Zhelyazkov, \& T. Mishonov (Melville, NY: AIP), 106

Zaqarashvili, T. V., Vörös, Z., \& Zhelyazkov, I. 2014, A\&A, 561, A62 